\title{
The Pressure Gap for Thiols: Methanethiol Self-Assembly on Au(111) from Vacuum to 1 bar
}

\author{
Rik V. Mom, ${ }^{*, \dagger, \bullet \wedge}$ Sigismund T. A. G. Melissen, ${ }^{\dagger, \bullet \wedge}{ }^{\wedge}$ Philippe Sautet, ${ }^{\S, \|_{\odot}}$ Joost W. M. Frenken, ${ }^{\perp}$
} Stephan N. Steinmann, ${ }^{*, \# \odot ~ a n d ~ I r e n e ~ M . ~ N . ~ G r o o t ~}{ }^{\dagger, \nabla} \nabla_{(}$

${ }^{\dagger}$ Huygens-Kamerlingh Onnes Laboratory, Leiden University, Niels Bohrweg 2, 2333 CA Leiden, The Netherlands

${ }^{\ddagger}$ Université de Lyon, Université Claude Bernard Lyon 1, CNRS, Institut Lumière Matière, F-69622 Lyon, France

${ }^{\S}$ Department of Chemical and Biomolecular Engineering and "Department of Chemistry and Biochemistry, University of California at Los Angeles, Los Angeles, California 90095, United States

${ }^{\perp}$ Advanced Research Center for Nanolithography, Science Park 110, 1098 XG Amsterdam, The Netherlands

${ }^{\#}$ Université de Lyon, Ens de Lyon, CNRS UMR 5182, Université Claude Bernard Lyon 1, Laboratoire de Chimie, F-69342, Lyon, France

${ }^{\nabla}$ Leiden Institute of Chemistry, Leiden University, Einsteinweg 55, 2333 CC Leiden, The Netherlands

Supporting Information

\begin{abstract}
Functionalizing noble metal surfaces with (bio)organic molecules is a vibrant field of research, with key applications in medicine, catalysis, and molecular electronics. Control over the molecular self-assembly is essential to creating functional devices. Here, we exploit our high-pressure, hightemperature scanning tunneling microscope (STM) to relate the effects of controllable parameters (temperature and pressure) to atomic-scale assembly mechanisms. Using methanethiol selfassembly on $\mathrm{Au}(111)$ as a model system, we monitor the formation and assembly of the ubiquitous $\left(\mathrm{CH}_{3} \mathrm{~S}\right)_{2} \mathrm{Au}$ "staple" motif into row structures at pressures of up to 1 bar. We observe a pressure-induced transition from the usual $1 / 3$ monolayer (ML) saturation coverage in vacuum to $3 / 8 \mathrm{ML}$ at $1 \mathrm{bar}$, thus providing the first evidence for a pressure gap effect for thiol adsorption. Complementing our experiments, we employed dispersion-corrected density functional theory computations to model the formed surface adlayers, corresponding STM images, and underlying equilibrium thermodynamics.
\end{abstract}

\section{INTRODUCTION}

Thiol molecules on gold provide a scaffold to which virtually any organic molecule can be attached. While the sulfur headgroup ensures firm bonding to the gold substrate, the tail of the molecule can be functionalized with reactive groups that allow bonding to other molecules. In this way, complex structures can be created that specifically target biomolecules in medical science (cf. refs 17-21 in ref 1), enhance (chiral) selectivity in catalysis, ${ }^{2}$ or provide unique electrical properties for molecular electronics. ${ }^{3}$ For these applications, control over the thiol surface structure is essential. At first glance, this task appears to be simple: thiol molecules on gold tend to form crystalline self-assembled monolayers (SAMs) (an overview of acronyms used in this manuscript is provided before the bibliography), even at moderate temperatures. ${ }^{1,2,4,5}$ However, the coexistence of multiple phases is difficult to prevent, particularly for larger thiol molecules. $4,6,7$

To find handles to tune the thiol overlayer structure, intimate knowledge of the bonding behavior and kinetics during self-assembly is required. A wide variety of microscopy, ${ }^{4,7-14}$ spectroscopy, ${ }^{15-20}$ and scattering ${ }^{21}$ techniques have been applied to these systems, complemented by theoretical modeling. ${ }^{1,19,21-23}$ It was found that following thiol adsorption the $\mathrm{S}-\mathrm{H}$ bond of the headgroup is broken and $\mathrm{H}_{2}$ is formed as a byproduct. While formally a thiyl radical is formed in this process, the adsorbate is usually called a "thiolate" or thiol, despite having neither a net negative charge nor a hydrogen atom. Thiolates have such an affinity for gold that $\mathrm{Au}$ atoms are extracted ${ }^{24}$ from the close-packed terraces to be incorporated in the thiol overlayer structure. ${ }^{1,9-11,21}$ It was established that the $\mathrm{Au}$ adatoms are bound by two thiol molecules, forming thiol-Au-thiol "staples". ${ }^{1,4,10,21}$ In the final step of overlayer formation, the staples self-assemble into crystalline phases.

While the fundamental studies have thus identified the key formation steps and structural elements in self-assembled monolayers, most of them were conducted under vacuum conditions, whereas elevated pressure or liquid-phase SAM

Received: April 1, 2019

Revised: April 24, 2019

Published: April 25, 2019 

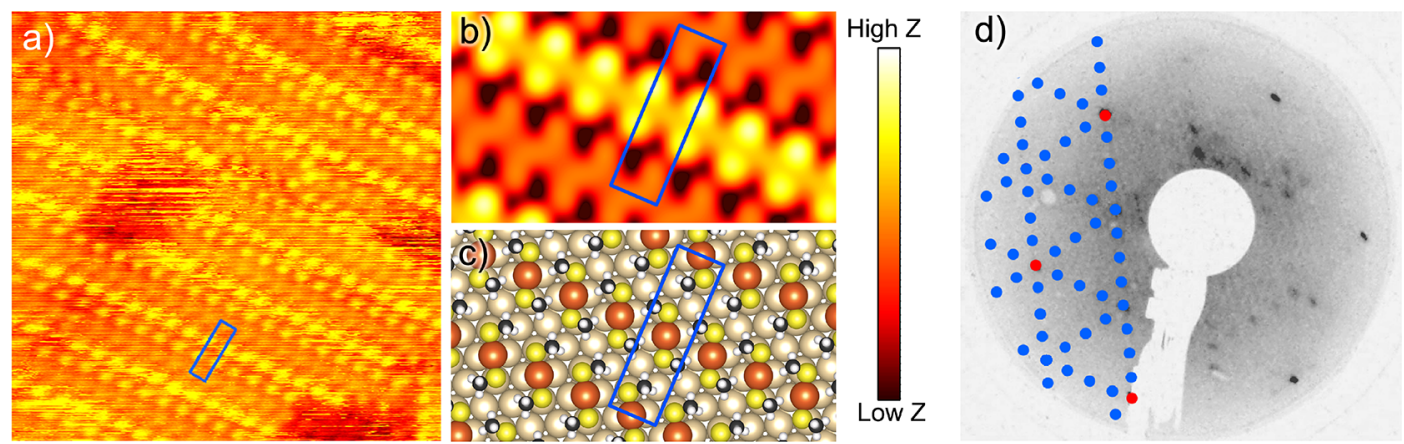

Figure 1. Adsorption structure of methanethiolate on $\mathrm{Au}(111)$ after saturation in vacuum. (a) $6 \times \sqrt{3}$ methanethiolate SAM obtained after $21 \mathrm{~h}$ of dosing $\left(P_{\mathrm{CH}_{3} \mathrm{SH}}=10^{-6} \mathrm{mbar}, P_{\mathrm{H}_{2}}=10^{-10} \mathrm{mbar}\right)$ at room temperature, $10 \times 10 \mathrm{~nm}^{2}, U_{\mathrm{s}}=-1.0 \mathrm{~V}, I_{\mathrm{t}}=150 \mathrm{pA}$. Corrugation in the SAM structure is $0.05 \mathrm{~nm}$, as determined from line scans perpendicular to the row direction. Note that the corrugation in STM images is strongly affected by the tip apex structure and might therefore change from image to image. (b) Simulated STM image ( $-1 \mathrm{~V}, 500 \mathrm{pA}$, corrugation $0.07 \mathrm{~nm})$ for the structure in panel a. (c) The corresponding ball model. Atoms in orange, Au adatoms; gold: lattice Au atoms; yellow, S; black, C; and white, H. (d) LEED pattern showing the $(1 \times 1)$ Au lattice (red dots) and the $6 \times \sqrt{3}$ SAM overlayer (blue dots, three domain orientations).

growth is much more attractive in practical applications. For several metal-adsorbate systems, it was shown that such a "pressure gap" can have a significant effect on the behavior of adsorbates. ${ }^{25-27}$ Here, we have studied methanethiol adsorption on $\mathrm{Au}(111)$ on both sides of the pressure gap: with our high-pressure, high-temperature scanning tunneling microscope (STM), ${ }^{28}$ we assess the SAM structures formed at pressures ranging from ultrahigh vacuum (UHV) to 1 bar at temperatures of up to $523 \mathrm{~K}$. Through the variation of temperature and pressure, we relate control parameters to the kinetic and thermodynamic factors that affect the SAM ordering and crystal structure. Our data is compared to extensive density functional theory (DFT) modeling, taking into account the effects of entropy and Van der Waals (VdW) interactions. This methodology allows us to thoroughly characterize SAM formation on the thermodynamic and kinetic levels under relevant conditions and characterize the highest-ever reported thiol coverage, here achieved at $1 \mathrm{bar}$ and $383 \mathrm{~K}$.

\section{METHODS}

2.1. Experimental Methods. 2.1.1. Setup Description. Microscopy at elevated pressure and temperature constitutes a technical challenge. To enable our studies at pressures of up to 1 bar, we used the ReactorSTM developed in our group. ${ }^{28}$ In short, this system allows for the preparation and characterization in UHV as well as high-pressure STM studies using a $0.5 \mathrm{~mL}$ flow cell inside the vacuum system. Gases are introduced into the flow reactor from a dedicated gas supply system, which allows for accurate control over the gas composition, flow, and pressure. All components that are in contact with the gas environment are made of corrosionresistant steel alloys, glass, poly(ether imide), or Kalrez to prevent degradation upon exposure to reactive gases such as $\mathrm{CH}_{3} \mathrm{SH}$. The use of PtIr STM tips prevents tip degradation during the measurements. Nevertheless, frequent changes in imaging quality due to the adsorption/desorption of gas molecules on the tip apex cannot be avoided.

In addition to the scanning tunneling microscope, the system is equipped with a low-energy electron diffraction (LEED) apparatus (Omicron) and an X-ray photoelectron spectrometer (SPECS Phoibos). A quadrupole mass spectrometer was used to verify the purity of the employed gases.
2.1.2. Experimental Procedures. The Au(111) sample (Surface Preparation Laboratories) $^{29}$ was cleaned by cycles of $1 \mathrm{keV} \mathrm{Ar}{ }^{+}$bombardment and annealing at $900 \mathrm{~K}$. For the formation of methanethiolate SAMs under UHV conditions, $\mathrm{CH}_{3} \mathrm{SH}$ (N1.8, Westfalen AG, containing approximately 2 vol $\%$ dimethyldisulfide (DMDS $)^{30}$ ) was dosed via capillaries close to the sample surface. All UHV dosing was performed at room temperature.

Prior to high-pressure experiments, all gas lines were flushed with argon for at least $30 \mathrm{~min}$. To start the high-pressure exposure, the reactor was slowly pressurized in argon or nitrogen before heating to the desired temperature. To minimize the thermal drift in the microscope, the system was allowed an equilibration period of approximately $90 \mathrm{~min}$. Whenever expedient, imaging was started before introducing $\mathrm{CH}_{3} \mathrm{SH}$ so that the surface dynamics upon exposure could be monitored.

2.2. Computational Methods. 2.2.1. Structure Determination. Electronic structure computations were performed using the Projector Augmented-Wave (PAW) formalism to account for the ion-electron interaction ${ }^{31}$ as implemented in Vienna Ab Initio Simulation Package (VASP) 5.4.1. ${ }^{32-35}$ The wave function was expanded in a plane wave basis set characterized by a cutoff energy of $400 \mathrm{eV}$. Generalized Gradient Approximation (GGA) functional Perdew-Burke-Ernzerhof $(\mathrm{PBE})^{36,37}$ was used, in combination with the "dDsC" dispersion correction, ${ }^{38}$ which has been shown to be accurate for adsorption energies on $\operatorname{Pt}(111)^{39}$ and has been successfully applied to the adsorption of pyridine on $\mathrm{Au}(111) .^{40}$ Second-order Methfessel-Paxton electron smearing (width of $0.2 \mathrm{eV}$ ) was applied to metallic surfaces. Ionic relaxations were performed using a conjugate-gradient algorithm to a maximum gradient below $0.05 \mathrm{eV} / \AA$, with wave functions converged to $10^{-5}$. A Monkhorst-Pack K-point sampling $^{41}$ of at least $3 \times 3 \times 1$ was applied to all surfaces in order to converge the adsorption energies. A primitive unit cell parameter of $2.928 \AA$ was specified, corresponding to the optimized Au bulk unit cell of $4.141 \AA$. A vacuum layer of $10 \AA$ was used to separate the vertical repetitions of the slabs.

For the STM simulations, the unit cell was replicated because our version of the code does not support K-point sampling. We used five full metallic layers, of which the three bottom layers were fixed in their bulk positions. Note that the 

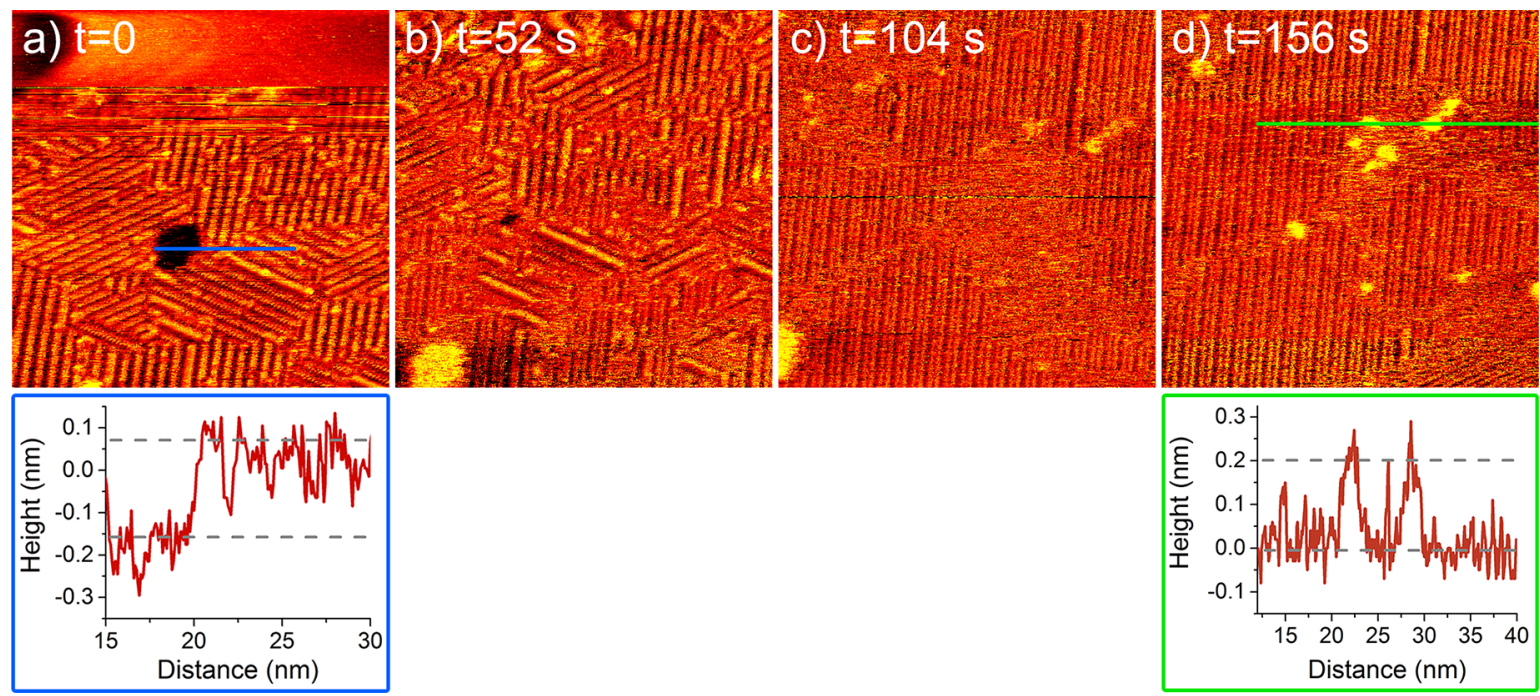

Figure 2. Structural transition from the $1 / 3 \mathrm{ML}$ phase to a high-pressure structure after introducing 1 bar $\mathrm{CH}_{3} \mathrm{SH}$ at $383 \mathrm{~K}$. Imaging parameters for $\mathrm{a}-\mathrm{d}$ : $40 \times 40 \mathrm{~nm}^{2}, U_{\mathrm{s}}=-1 \mathrm{~V}, I_{\mathrm{t}}=150 \mathrm{pA}$. Height lines identify an Au vacancy island (blue) and Au adatom islands (green).

herringbone reconstruction ${ }^{42}$ was neglected in our computations.

STM images were simulated by the approach of Tersoff and Hamann, ${ }^{43-45}$ at a bias voltage of $-1 \mathrm{~V}$ and a current of $10^{-9} \mathrm{~A}$ (unless stated otherwise) on the basis of the PBE band structure at the $\Gamma$ point of super cells.

2.2.2. Thermodynamics. Frequency computations were performed to estimate zero-point energies and vibrational entropy corrections within the harmonic oscillator approximation. For these computations, all gold atoms were kept frozen. All frequencies were real and above $40 \mathrm{~cm}^{-1}$. Adsorption energies for structures with gold adatoms are referenced to the $\mathrm{Au}(111)$ surface and $\mathrm{Au}$ atoms from the bulk. Adsorption free energies were calculated on the basis of the following reaction:

$$
\mathrm{CH}_{3} \mathrm{SH}+\mathrm{Au}(111) \rightleftharpoons \mathrm{CH}_{3} \mathrm{~S}-\mathrm{Au}(111)+\frac{1}{2} \mathrm{H}_{2}
$$

When estimating thermal contributions to the Gibbs energy, we made the usual assumptions of harmonic oscillators for vibrations. For gas-phase molecules, we also invoke the rigidrotor approximation and the Sackur-Tetrode equation for the rotational and translational contributions, respectively. The energy required to bring one $\mathrm{Au}$ atom from the bulk onto the $\mathrm{Au}(111)$ surface is on the order of $0.7 \mathrm{eV}$. To allow for a wider comparison of our data to the literature, we also provide data for the calculation of adsorption energies in a DMDS gas atmosphere in Table S1 in the SI.

\section{RESULTS AND DISCUSSION}

We investigated the effect of pressure on the structure of the methanethiolate overlayer using controlled dosing experiments in vacuum $\left(10^{-6} \mathrm{mbar}\right)$ and at 1 bar. For the vacuum case, several saturation structures have already been identified in the literature, ${ }^{11,46,47}$ each with $1 / 3$ monolayer (ML) coverage. In our case, the surface is saturated with a striped phase with a $6 \times \sqrt{3}$ unit cell (Figure 1) after several hours of $10^{-6}$ mbar $\mathrm{CH}_{3} \mathrm{SH}$ exposure at room temperature. (See Figure $\mathrm{S} 1$ in the ESI for the structural evolution during the self-assembly.) Similar striped phases have been reported in the literature, although ordering of the stripes into densely packed domains was never observed. ${ }^{10,11,46,48}$ We point out that exchange reactions with strong-binding impurities from the gas feed, such as ethanethiol, can be excluded because the stripe motifs were already observed in the initial stages of self-assembly (90 min, Figure S1a in the SI). The stripes have been identified as rows of thiol-Au-thiol staples, which can appear in several structural isomers. Our DFT simulations (SI section S3.3) of the staple structures indicate that the inclusion of $\mathrm{Au}$ adatoms in the SAMs indeed improves the Au-S interaction $(\sim 0.25 \mathrm{eV}$ per thiol), while the staple packing and isomeric form are determined by the methyl-methyl repulsion (up to $\sim 0.4 \mathrm{eV}$ per thiol). Our computations indicate that several isomers have a very comparable formation energy, explaining why multiple phases have been observed in the literature..$^{10,11,46,47}$ Indeed, depending on the tip's state and its interactions with the surface, the SAM was sometimes observed as a $3 \times \sqrt{3}$ line structure (Figure S2 in the SI).

To identify the experimentally observed structure observed in Figure 1a, we used simulated STM images. The simulated STM image in Figure $1 b$ (with the corresponding ball model in Figure 1c) shows the best agreement with the experimental image (Figure 1a), accurately reproducing the alternating brightness in the line structure, in contrast to the simulations of other models (Figure S3 in the SI). Although the fine structure is not fully resolved in the experimental image, the bright protrusions $(\sim 0.1-0.2 \AA)$ originating from half of the methyl groups show the same rectangular pattern in both the experimental and simulated images. With this good agreement, the structure in Figure $1 \mathrm{c}$ is assigned to the vacuum saturation coverage structure shown in Figure 1a. Similar to the other phases observed in the literature, ${ }^{11,46,47}$ this structure has a coverage of $1 / 3 \mathrm{ML}$.

To investigate if a coverage higher than $\frac{1}{3} \mathrm{ML}$ can be obtained at elevated pressure, we imaged the $\mathrm{Au}(111)$ surface in 1 bar $\mathrm{CH}_{3} \mathrm{SH}$ at several temperatures. Figure 2 shows the structural evolution of the SAM directly after changing the partial pressure of $\mathrm{CH}_{3} \mathrm{SH}$ from trace levels to 1 bar at $383 \mathrm{~K}$. The initial vacuum saturation-coverage phase (which appears as a $3 \times \sqrt{3}$ structure in Figure $2 a$ ) is quickly replaced by a new phase with paired lines. The new structure is stable only under 
high-pressure conditions: when the partial pressure of $\mathrm{CH}_{3} \mathrm{SH}$ is brought back down to trace levels, the $3 \times \sqrt{3}$ structure is recovered (Figure 3). Thus, it is clear that the $\mathrm{CH}_{3} \mathrm{SH}$ pressure can be used to drive a reversible phase transition.

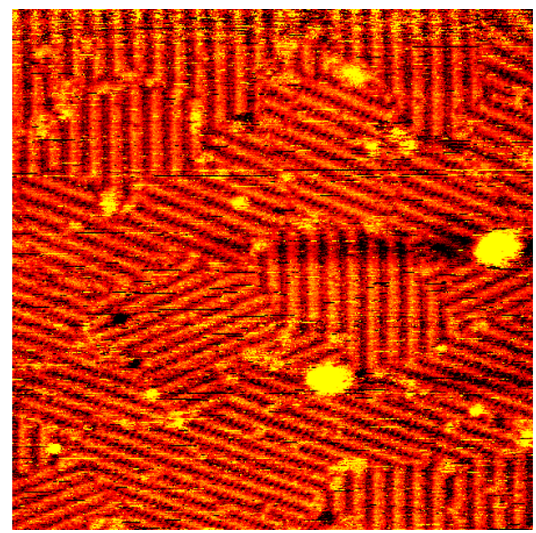

Figure 3. Reversibility of the high-pressure phase transition following the decrease in the $\mathrm{CH}_{3} \mathrm{SH}$ pressure to trace levels (in 1 bar $\mathrm{N}_{2}$ ), 383 K. Imaging parameters: $35 \times 35 \mathrm{~nm}^{2}, U_{\mathrm{s}}=-1 \mathrm{~V}, I_{\mathrm{t}}=150 \mathrm{pA}$.

For such a pressure-driven transition, there is an increase in the adsorbate coverage. ${ }^{25-27}$ To establish which $\mathrm{CH}_{3} \mathrm{SH}$ coverage is achieved, we first determined the unit cell shape under high-pressure conditions. This is a challenge because the drift in the microscope at high pressure and temperature causes some image distortion. Using large-scale images with a high tip speed, we minimized the effect of drift and established that the unit cell has the same orientation as in the 1/3 ML case. The periodicity perpendicular to the stripes is four $\mathrm{Au}$ spacings. The periodicity along the stripe direction was determined to be $\sqrt{3}$ Au spacing using Figure $4 c$. Thus, we arrive at a $4 \times \sqrt{3}$ unit cell. The highest thiol coverage that allows for reasonable orientation of the methyl groups in this unit cell is $3 / 8 \mathrm{ML}$.

A closer inspection of the images in Figure 2 reveals that the denser packing of $\mathrm{CH}_{3} \mathrm{SH}$ is enabled by the release of $\mathrm{Au}$ from the SAM: the vacancy island in Figure $2 \mathrm{a}$ is filled (Figure $2 \mathrm{c}$ ), and $\mathrm{Au}$ adatom islands (Figure $2 \mathrm{~b}-\mathrm{d}$ ) are formed. We quantified the vacancy island coverage within a few minutes after the transition to high pressure in several fresh areas on wide terraces (e.g., Figure 4a), thus minimizing the influence of the tip and step edges as sinks for the adatoms. We find that the amount of released $\mathrm{Au}$ is large and strongly fluctuating: $0.22 \pm 0.07 \mathrm{ML}$. This amount is in the same range as the 0.17 ML that is contained in the perfect $1 / 3 \mathrm{ML}$ structure. On the basis of this, one could hypothesize that the high-pressure phase does not contain $\mathrm{Au}$ adatoms. However, our DFT computations show that $3 / 8 \mathrm{ML}$ structures without $\mathrm{Au}$ adatoms are more than $0.2 \mathrm{eV}$ less stable per adsorbed thiol (i.e., more than $0.6 \mathrm{eV}$ for the $3 / 8 \mathrm{ML}$ unit cell) than their Aucontaining counterparts. (See Table S2 in the SI for the most stable one out of seven tested configurations.) Furthermore, the large spread in Au adatom island coverage suggests that there is a second source of $\mathrm{Au}$ atoms that shows more fluctuation than a pure crystalline phase. We suggest that the domain boundaries in the SAM are this second source. Indeed, Figure 3 shows that small Au clusters can be generated at the domain boundaries following dynamic situations such as phase transitions or the initial formation of the SAM.

On the basis of the discussion above, we considered candidate high-pressure structures with a $4 \times \sqrt{3}$ unit cell, 3/ $8 \mathrm{ML} \mathrm{CH}_{3} \mathrm{SH}$ coverage, and $1 / 8 \mathrm{ML} \mathrm{Au}$ adatom coverage (Figure S4 in the SI). Following the structural motifs identified in the literature, these contain a mixture of "staples" and bridge-bonded thiolates. ${ }^{10,11,21,46,47,49}$ Again, we find several isomers with similar formation energy, necessitating further distinction using STM simulations. The simulation in Figure $4 \mathrm{~d}$, based on the structure in Figure $4 \mathrm{e}$, reproduces the bright paired rows observed in the experiment (Figure 4c). Note that the row spacing in the experimental image (Figure 4c) is somewhat stretched by the drift in the microscope under highpressure, high-temperature conditions. The "bright" row pairs, also clearly observed for the low-pressure case (Figure 1a,b), characterize the trans-staple motif for which the methyl groups are equidistant to (1) the $\mathrm{Au}$ adatom to which they are covalently bound through $S$ and (2) a neighboring Au adatom. The thiolates between the staples, which are bridge-bonded with a tendency toward the hcp site, appear dark in both the experimental and simulated image. The alignment of the bridge-bonded thiolates with respect to the staples is difficult to determine from the experimental image, but the STM simulations allow differentiation (cf. Figure S4) between very similar structures. On the basis of this agreement, we identify the high-pressure structure with the structure shown in Figure $4 \mathrm{e}$.
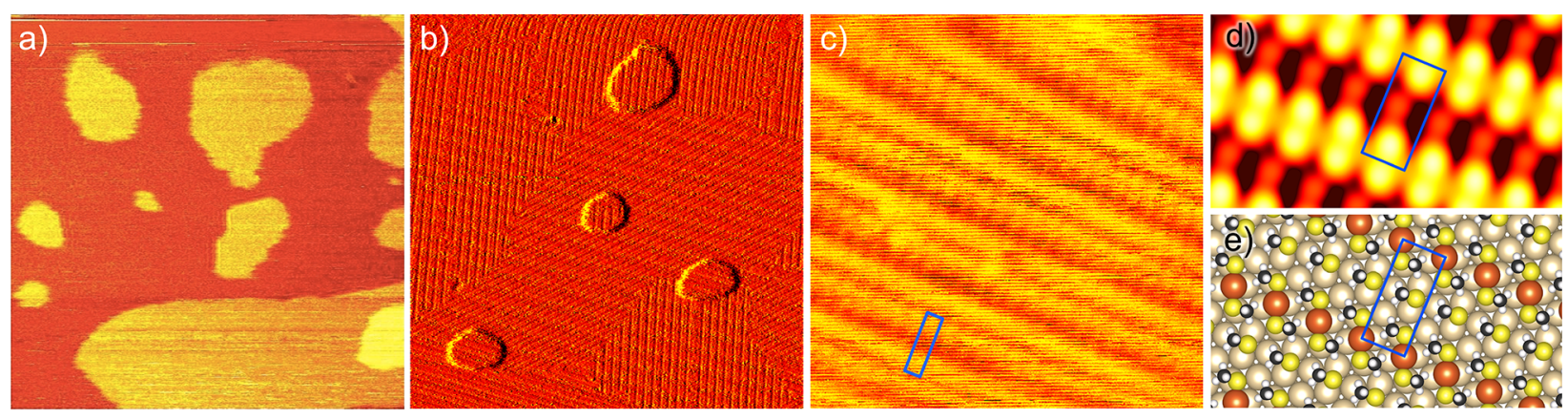

Figure 4. Structure of methanethiolate $\mathrm{SAM}$ at $1 \mathrm{bar}\left(P_{\mathrm{CH}_{3} \mathrm{SH}}=1000 \mathrm{mbar}, P_{\mathrm{H}_{2}}=0.35 \mathrm{mbar}\right), 383 \mathrm{~K}$. (a) Au adatom islands shortly after the transition from trace levels to $1 \mathrm{bar} \mathrm{CH}_{3} \mathrm{SH}\left(80 \times 80 \mathrm{~nm}^{2}, U_{\mathrm{s}}=-0.2 \mathrm{~V}, I_{\mathrm{t}}=120 \mathrm{pA}\right)$. (b) SAM structure on adatom islands. The STM image was differentiated to visualize the SAM structure on both the terrace and the adatom islands $\left(80 \mathrm{~nm} \times 80 \mathrm{~nm}, U_{\mathrm{s}}=-0.1 \mathrm{~V}, I_{\mathrm{t}}=800 \mathrm{pA}\right)$. (c) Details of the SAM structure, $10 \times 10 \mathrm{~nm}^{2}, U_{s}=-0.1 \mathrm{~V}, I_{\mathrm{t}}=800 \mathrm{pA}$. Corrugation in SAM structure: $0.02 \mathrm{~nm}$. (d) Simulated STM image $(-1 \mathrm{~V}, 500 \mathrm{pA}$, corrugation $0.08 \mathrm{~nm}$ ) for the structure in panel c. (e) The corresponding ball model. 
With a coverage of $3 / 8 \mathrm{ML}$, this is the most densely packed SAM reported in the literature, evidencing the existence of a pressure gap for thiol adsorption. Note that Mehring et al. ${ }^{14}$ observed a similar $4 \times \sqrt{3}$ structure after the exposure of $\mathrm{Au}(111)$ to a $1 \mathrm{mM} \mathrm{DMDS} / \mathrm{ethanol}$ solution for several hours. They suggested a structure with only 1/4 ML coverage. While the competition between thiol and solvent adsorption might have resulted in such a structure under their conditions, our observation of a pressure-induced transition provides clear evidence of a structure with a coverage higher than that obtained in UHV.

To relate the observations in Figures $1 \mathrm{a}$ and $4 \mathrm{c}$ to equilibrium thermodynamics, we calculated the corresponding adsorption free energies using

$$
\Delta G_{\mathrm{ads}}=\Delta E_{\mathrm{ads}}+\Delta \mathrm{ZPE}_{\mathrm{ads}}+\delta H_{\mathrm{ads}}^{T}-T \Delta S_{\mathrm{ads}}
$$

where $E_{\mathrm{ads}}$ is the sum of electronic energies $E_{\mathrm{PBE}}$ and $E_{\mathrm{dDs} C}$, $\mathrm{ZPE}$ refers to the zero-point energies, $\delta H^{T}$ refers to the thermal correction to the enthalpy, and $S$ refers to the entropy. The vibrational contributions of the SAMs were taken into account in the calculation (details in the Thermodynamics section).

Figure 5 shows that both the $1 / 3 \mathrm{ML}$ and $3 / 8 \mathrm{ML}$ structures are thermodynamically predicted to be at least metastable at

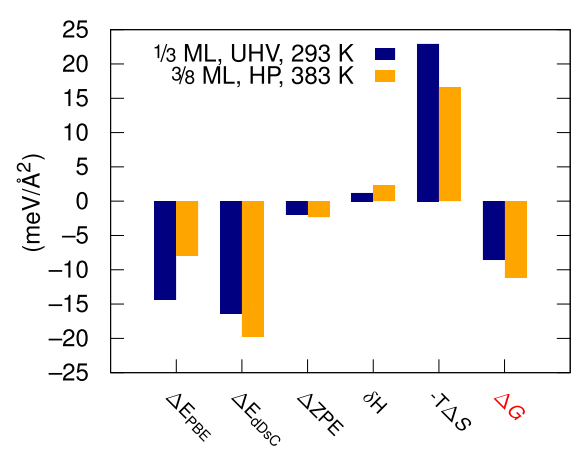

Figure 5. Contributions to the surface free energy according to eq 2 for $1 / 3 \mathrm{ML}$ under UHV, $293 \mathrm{~K}$ and $3 / 8 \mathrm{ML}$ under $\mathrm{HP}, 383 \mathrm{~K}$ conditions.

the respective conditions under which they were experimentally observed. (See Section 3.4 in the ESI for a discussion on relative stability.) Second, the decomposition shows that neither the change in ZPE, nor in the enthalpy corrections contributes significantly to the adsorption free energy. The adsorption entropy, however, does significantly offset $\Delta E_{\text {ads }}$. Because the entropic contribution to the free energy is strongly dependent on temperature and pressure, this shows that the applied conditions strongly influence the stability of the SAM structures. The pressure increase from $10^{-6} \mathrm{mbar}$ to $1 \mathrm{bar}$ lowers the entropic penalty for adsorption according to

$$
\Delta G=k_{\mathrm{B}} T \ln \left(p / p^{\prime}\right)
$$

Despite the $90 \mathrm{~K}$ temperature difference between Figures 1a and $4 \mathrm{c}$, Figure 5 shows that the pressure increase dominates and thus lowers the entropy cost, providing a stabilizing force for higher thiol coverage. A further temperature increase should undo this stabilizing effect, however, and can even lead to desorption of the SAM. Figure 6a shows that desorption occurs at $523 \mathrm{~K}$, where only the $(1 \times 1) \mathrm{Au}(111)$ lattice is visible despite the high $\mathrm{CH}_{3} \mathrm{SH}$ pressure. Note that the observation of the $(1 \times 1)$ lattice does not imply a completely empty surface because a small amount of thiolates could be obscured from STM observation as a result of their fast motion. Indeed, the absence of the heringbone reconstruction indicates that some thiolates remain on the surface. Nonetheless, the coverage decrease demonstrates that the entropy in the gas phase outcompetes both the pressure and the adsorption energy under these conditions.

At lower temperatures, adsorption kinetics play a dominant role. As can be seen in Figure $6 \mathrm{~b}$, room-temperature exposure at 1 bar results in a very poorly ordered structure, containing $3 \times \sqrt{3}$ line structures similar to those observed after UHV exposure. The lack of ordering establishes that thiol adsorption, $\mathrm{S}-\mathrm{H}$ bond dissociation, and staple formation proceed significantly faster than the ordering process. Note that the SAM structure did not proceed to the $4 \times \sqrt{3}$ phase even though the thermodynamic driving force is higher than in the experiment at $383 \mathrm{~K}$ (Figure 4c). Clearly, the hampered diffusion at $1 / 3 \mathrm{ML}$ coverage prevents the insertion of more thiol molecules into the SAM.

On the basis of our observations and simulations for various conditions, Figure 7 shows an overview of the relation between the applied conditions and the observed structure. One distinguishes conditions where the structure is determined by kinetics and others that lead to thermodynamic control. The kinetically controlled region tends toward lower coverage and disorder and originates from the mismatch between the (fast) staple formation rate and the (slower) staple ordering rate. The
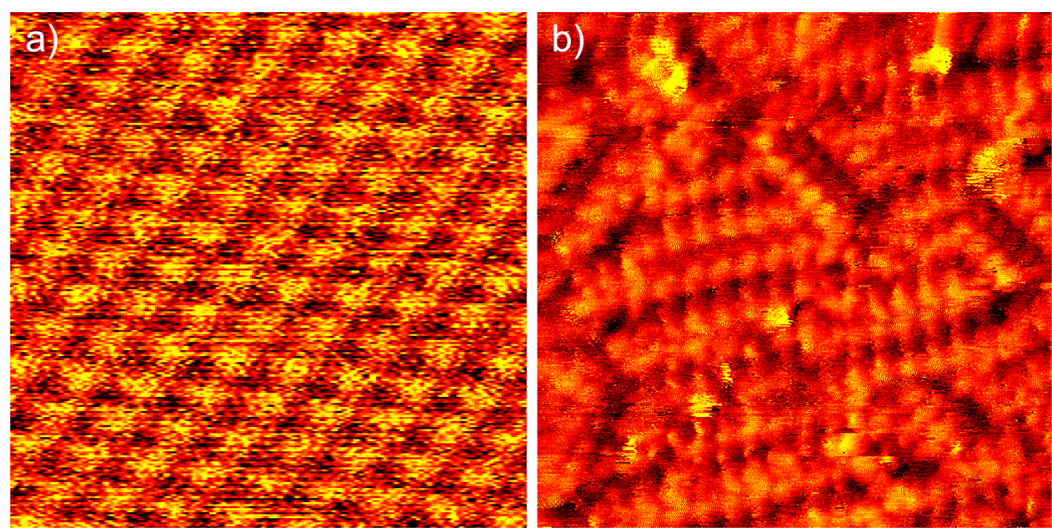

Figure 6. Effect of temperature on $\mathrm{CH}_{3} \mathrm{SH}$ adsorption at 1 bar. (a) Empty $\mathrm{Au}(111)$ surface imaged at $523 \mathrm{~K}\left(2.5 \times 2.5 \mathrm{~nm}^{2}, U_{\mathrm{s}}=-0.3 \mathrm{~V}, I_{\mathrm{t}}=520\right.$ $\mathrm{pA})$. (b) Kinetically hindered $\mathrm{CH}_{3} \mathrm{SH}$ adsorption at room temperature $\left(10 \times 10 \mathrm{~nm}^{2}, U_{\mathrm{s}}=-0.3 \mathrm{~V}, I_{\mathrm{t}}=520 \mathrm{pA}\right)$. 


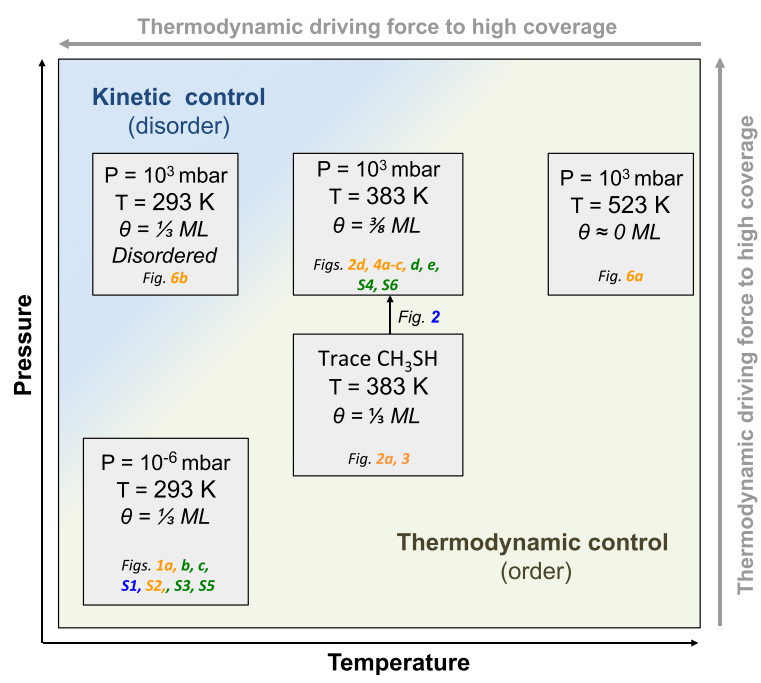

Figure 7. Conceptualization of thermodynamic and kinetic factors determining SAM structures. The different structures and transformations are linked to the figures in this article. (Blue) SAM formation process. (Green) Theoretical analysis. (Orange) Equilibrium/kinetically stable SAMs.

rate mismatch is largest for low temperature (Figure $6 \mathrm{~b}$ vs Figure $4 c$ ) and/or high pressure (Figure $6 \mathrm{~b}$ vs Figure 1a).

At low pressure and/or high temperature, thermodynamic control takes over. Our computations show that the balance of the $\mathrm{Au}-\mathrm{S}$ interaction, the van der Waals interactions, and the gas-phase entropy determines the relative stability of the various SAM structures. Through the gas-phase entropy, higher pressure favors higher coverage (Figure $4 \mathrm{c}$ vs Figure 1a), while higher temperature favors lower coverage (Figure $4 \mathrm{c}$ vs Figure 6). Note, however, that our computations show that several structures with the same coverage are energetically very close (Figures S3-S6 in the SI). Thus, subtle factors such as the energetic cost of defect formation (impurities, step edges, and domain boundaries) may also determine which structure is observed.

\section{CONCLUSIONS}

We have identified the key thermodynamic and kinetic factors that control methanethiolate SAM formation and have related these factors to the applied temperature and pressure. Most importantly, we have shown that, beside affecting the SAM order, the dosing pressure can induce a phase transition to the newly revealed $3 / 8$ ML phase. Such pressure- or concentration-induced transitions will likely also occur for functionalized SAMs, for which repulsive tail-tail interactions may exist, which are in competition with optimizing the Au-thiol interaction. ${ }^{50} \mathrm{We}$ therefore expect that the fundamental insights gained here will apply to a wide range of technologically relevant SAMs and provide handles for their controlled growth.

\section{ASSOCIATED CONTENT}

\section{Supporting Information}

The Supporting Information is available free of charge on the ACS Publications website at DOI: 10.1021/acs.jpcc.9b03045.

Additional STM data and an extensive theoretical analysis based on DFT energetics and simulated STM images; an expression for the equilibrium hydrogen pressure in the reactor; the geometries of all structures in the CONTCAR format; and the energies and entropies computed for the $\mathrm{ab}$ initio thermodynamics modeling are provided (PDF)

\section{AUTHOR INFORMATION}

\section{Corresponding Authors}

*E-mail: mom@physics.leidenuniv.nl. Phone: +49.30.8413.4640. Fax: +49.30.8413.4401.

*E-mail: stephan.steinmann@ens-lyon.fr. Phone: + 33.4.72.72.81.55. Fax: + 33.4.72.72.88.60.

ORCID $\odot$

Rik V. Mom: 0000-0002-5111-5591

Sigismund T. A. G. Melissen: 0000-0002-8728-9382

Philippe Sautet: 0000-0002-8444-3348

Stephan N. Steinmann: 0000-0002-2777-356X

Irene M. N. Groot: 0000-0001-9747-3522

\section{Present Address}

-Fritz-Haber Institut der Max-Planck-Gesellschaft, Faradayweg 4-6, 14195 Berlin, Germany

\section{Author Contributions}

${ }^{\wedge}$ These authors contributed equally to this article.

\section{Notes}

The authors declare no competing financial interest.

\section{ACKNOWLEDGMENTS}

This project was financially supported by a Dutch SmartMix grant and by NIMIC partner organizations through NIMIC, a public-private partnership. I.M.N.G. acknowledges the Dutch organization for scientific research (NWO-STW) for her Veni fellowship. S.N.S. and S.T.A.G.M. gratefully acknowledge the computational resources provided by the Pôle Scientifique de Modélisation Numérique (PSMN) at ENS Lyon. S.N.S. is grateful to N. Lorente and T. Jiang for sharing code and scripts, respectively.

\section{LIST OF ALPHABETICALLY ORDERED ACRONYMS}

DFT density functional theory; DMDS dimethyldisulfide; GGA generalized gradient approximation; hcp hexagonally close packed; LEED low-energy electron diffraction; ML monolayer; PAW projector-augmented wave; PBE PerdewBurke-Ernzerhof; PES potential energy surface; SAM selfassembled monolayers; STM scanning tunneling microscope; UHV ultrahigh vacuum; VASP Vienna ab initio simulation package; vdW van der Waals; ZPE zero-point energy

\section{REFERENCES}

(1) Häkkinen, H. The Gold-Sulfur Interface at the Nanoscale. Nat. Chem. 2012, 4, 443-455.

(2) Bürgi, T. Properties of the Gold-Sulphur Interface: from SelfAssembled Monolayers to Clusters. Nanoscale 2015, 7, 15553-15567.

(3) Tour, J. M. Molecular Electronics. Synthesis and Testing of Components. Acc. Chem. Res. 2000, 33, 791-804.

(4) Pensa, E.; Cortés, E.; Corthey, G.; Carro, P.; Vericat, C.; Fonticelli, M. H.; Benitez, G.; Rubert, A. A.; Salvarezza, R. C. The Chemistry of the Sulfur-Gold Interface: In Search of a Unified Model. Acc. Chem. Res. 2012, 45, 1183-1192.

(5) Poirier, G. E. Characterization of Organosulfur Molecular Monolayers on $\mathrm{Au}$ (111) Using Scanning Tunneling Microscopy. Chem. Rev. 1997, 97, 1117-1128.

(6) Sotthewes, K.; Wu, H.; Kumar, A.; Vancso, G. J.; Schön, P. M.; Zandvliet, H. J. W. Molecular Dynamics and Energy Landscape of 
Decanethiolates in Self-Assembled Monolayers on $\mathrm{Au}(111)$ Studied by Scanning Tunneling Microscopy. Langmuir 2013, 29, 3662-3667. (7) Li, F.; Tang, L.; Zhou, W.; Guo, Q. Adsorption Site Determination for Au-Octanethiolate on $\mathrm{Au}(111)$. Langmuir 2010, 26, 9484-9490.

(8) Dishner, M. H.; Hemminger, J. C.; Feher, F. J. Direct Observation of Substrate Influence on Chemisorption of Methanethiol Adsorbed from the Gas Phase onto the Reconstructed $\mathrm{Au}(111)$ Surface. Langmuir 1997, 13, 2318-2322.

(9) Kautz, N. A.; Kandel, S. A. Alkanethiol Monolayers Contain Gold Adatoms, and Adatom Coverage Is Independent of Chain Length. J. Phys. Chem. C 2009, 113, 19286-19291.

(10) Maksymovych, P.; Sorescu, D. C.; Yates, J. T. Gold-AdatomMediated Bonding in Self-Assembled Short-Chain Alkanethiolate Species on the $\mathrm{Au}(111)$ Surface. Phys. Rev. Lett. 2006, 97, DOI: 10.1103/PhysRevLett.97.146103.

(11) Voznyy, O.; Dubowski, J. J.; Yates, J. T.; Maksymovych, P. The Role of Gold Adatoms and Stereochemistry in Self-Assembly of Methylthiolate on $\mathrm{Au}(111)$. J. Am. Chem. Soc. 2009, 131, 1298912993.

(12) Zhang, J.; Chi, Q.; Ulstrup, J. Assembly Dynamics and Detailed Structure of 1-Propanethiol Monolayers on $\mathrm{Au}(111)$ Surfaces Observed Real Time by in situ STM. Langmuir 2006, 22, 6203-6213.

(13) Guo, Q.; Li, F. Self-Assembled Alkanethiol Monolayers on Gold Surfaces: Resolving the Complex Structure at the Interface by STM. Phys. Chem. Chem. Phys. 2014, 16, 19074-19090.

(14) Mehring, P.; Beimborn, A.; Westphal, C. The Structural Formation of Methylthiolate SAMs on $\mathrm{Au}(111)$ for Short Deposition Times from Solution. Appl. Surf. Sci. 2010, 256, 7265-7269.

(15) Roper, M. G.; Jones, R. G. Methylthiolate on Au(111): Adsorption and Desorption Kinetics. Phys. Chem. Chem. Phys. 2008, $10,1336-1346$

(16) Kondoh, H.; Iwasaki, M.; Shimada, T.; Amemiya, K.; Yokoyama, T.; Ohta, T.; Shimomura, M.; Kono, S. Adsorption of Thiolates to Singly Coordinated Sites on $\mathrm{Au}(111)$ Evidenced by Photoelectron Diffraction. Phys. Rev. Lett. 2003, 90, 066102.

(17) Rousseau, R.; De Renzi, V.; Mazzarello, R.; Marchetto, D.; Biagi, R.; Scandolo, S.; del Pennino, U. Interfacial Electrostatics of Self-Assembled Monolayers of Alkane Thiolates on $\mathrm{Au}(111)$ : Work Function Modification and Molecular Level Alignments. J. Phys. Chem. B 2006, 110, 10862-10872.

(18) Lavrich, D. J.; Wetterer, S. M.; Bernasek, S. L.; Scoles, G. Physisorption and Chemisorption of Alkanethiols and Alkyl Sulfides on $\mathrm{Au}$ (111). J. Phys. Chem. B 1998, 102, 3456-3465.

(19) Mazzarello, R.; Cossaro, A.; Verdini, A.; Rousseau, R.; Casalis, L.; Danisman, M. F.; Floreano, L.; Scandolo, S.; Morgante, A.; Scoles, G. Structure of a $\mathrm{CH}_{3} \mathrm{~S}$ Monolayer on $\mathrm{Au}(111)$ Solved by the Interplay between Molecular Dynamics Calculations and Diffraction Measurements. Phys. Rev. Lett. 2007, 98, 016102.

(20) Cheng, H.; Yang, L.; Jiang, Y.; Huang, Y.; Sun, Z.; Zhang, J.; Hu, T.; Pan, Z.; Pan, G.; Yao, T.; et al. Adsorption Kinetic Process of Thiol Ligands on Gold Nanocrystals. Nanoscale 2013, 5, 1179511800.

(21) Cossaro, A.; Mazzarello, R.; Rousseau, R.; Casalis, L.; Verdini, A.; Kohlmeyer, A.; Floreano, L.; Scandolo, S.; Morgante, A.; Klein, M. L.; et al. X-ray Diffraction and Computation Yield the Structure of Alkanethiols on Gold(111). Science 2008, 321, 943-946.

(22) Andersson, M. P. Density Functional Theory with Modified Dispersion Correction for Metals Applied to Self-Assembled Monolayers of Thiols on $\mathrm{Au}(111)$. J. Theor. Chem. 2013, 2013, 327839.

(23) Jiang, D.-e.; Dai, S. Cis-trans Conversion of the $\mathrm{CH}_{3} \mathrm{~S}-\mathrm{Au}-\mathrm{SCH}_{3}$ Complex on $\mathrm{Au}(111)$. Phys. Chem. Chem. Phys. 2009, 11, 8601-8605.

(24) Liao, W.-S.; Cheunkar, S.; Cao, H. H.; Bednar, H. R.; Weiss, P. S.; Andrews, A. M. Subtractive Patterning via Chemical Lift-Off Lithography. Science 2012, 337, 1517-1521.

(25) van Spronsen, M. A.; Frenken, J. W. M.; Groot, I. M. N. Observing the Oxidation of Platinum. Nat. Commun. 2017, 8, 429.
(26) Tao, F.; Dag, S.; Wang, L.-W.; Liu, Z.; Butcher, D.; Bluhm, H.; Salmeron, M.; Somorjai, G. Break-Up of Stepped Platinum Catalyst Surfaces by High CO coverage. Science 2010, 327, 850-853.

(27) Vang, R. T.; Lægsgaard, E.; Besenbacher, F. Bridging the Pressure Gap in Model Systems for Heterogeneous Catalysis with High-Pressure Scanning Tunneling Microscopy. Phys. Chem. Chem. Phys. 2007, 9, 3460-3469.

(28) Herbschleb, C.; van der Tuijn, P.; Roobol, S.; Navarro, V.; Bakker, J.; Liu, Q.; Stoltz, D.; Cañas-Ventura, M.; Verdoes, G.; van Spronsen, M.; et al. The ReactorSTM: Atomically Resolved Scanning Tunneling Microscopy under High-Pressure, High-Temperature Catalytic Reaction Conditions. Rev. Sci. Instrum. 2014, 85, 083703.

(29) Surface Preparation Laboratory, https://www.spl.eu/, consulted 2018-1-29.

(30) This is due to the factory preparation conditions of $\mathrm{CH}_{3} \mathrm{SH}$ and not to the equilibration reaction.

(31) Kresse, G.; Joubert, D. From Ultrasoft Pseudopotentials to the Projector Augmented-Wave Method. Phys. Rev. B: Condens. Matter Mater. Phys. 1999, 59, 1758-1775.

(32) Kresse, G.; Hafner, J. Ab Initio Molecular Dynamics for Liquid Metals. Phys. Rev. B: Condens. Matter Mater. Phys. 1993, 47, 558-561.

(33) Kresse, G.; Hafner, J. Ab initio Molecular-Dynamics Simulation of the Liquid-MetalAmorphous-Semiconductor Transition in Germanium. Phys. Rev. B: Condens. Matter Mater. Phys. 1994, 49, 1425114269.

(34) Kresse, G.; Furthmüller, J. Efficiency of Ab-Initio Total Energy Calculations for Metals and Semiconductors Using a Plane-Wave Basis Set. Comput. Mater. Sci. 1996, 6, 15-50.

(35) Kresse, G.; Furthmüller, J. Efficient Iterative Schemes for $A b$ Initio Total-Energy Calculations Using a Plane-Wave Basis Set. Phys. Rev. B: Condens. Matter Mater. Phys. 1996, 54, 11169-11186.

(36) Perdew, J. P.; Burke, K.; Ernzerhof, M. Generalized Gradient Approximation Made Simple. Phys. Rev. Lett. 1996, 77, 3865-3868.

(37) Perdew, J. P.; Burke, K.; Ernzerhof, M. Erratum: Generalized Gradient Approximation Made Simple [Phys. Rev. Lett. 77, 3865 (1996)]. Phys. Rev. Lett. 1997, 78, 1396-1396.

(38) Steinmann, S. N.; Corminboeuf, C. Comprehensive Benchmarking of a Density-Dependent Dispersion Correction. J. Chem. Theory Comput. 2011, 7, 3567-3577.

(39) Gautier, S.; Steinmann, S. N.; Michel, C.; Fleurat-Lessard, P.; Sautet, P. Molecular Adsorption at $\mathrm{Pt}(111)$. How Accurate are DFT Functionals? Phys. Chem. Chem. Phys. 2015, 17, 28921-28930.

(40) Steinmann, S. N.; Sautet, P. Assessing a First-Principles Model of an Electrochemical Interface by Comparison with Experiment. $J$. Phys. Chem. C 2016, 120, 5619-5623.

(41) Monkhorst, H. J.; Pack, J. D. Special Points for Brillouin-Zone Integrations. Phys. Rev. B 1976, 13, 5188-5192.

(42) Poirier, G. E.; Pylant, E. D. The Self-Assembly Mechanism of Alkanethiols on Au (111). Science 1996, 272, 1145-1147.

(43) Tersoff, J.; Hamann, D. R. Theory and Application for the Scanning Tunneling Microscope. Phys. Rev. Lett. 1983, 50, 19982001.

(44) Tersoff, J.; Hamann, D. R. Theory of the Scanning Tunneling Microscope. Phys. Rev. B: Condens. Matter Mater. Phys. 1985, 31, 805-813.

(45) Lorente, N.; Persson, M. Theory of Single Molecule Vibrational Spectroscopy and Microscopy. Phys. Rev. Lett. 2000, 85, 2997-3000.

(46) Tang, L.; Li, F.; Zhou, W.; Guo, Q. The Structure of Methylthiolate and Ethylthiolate Monolayers on $\mathrm{Au}(111)$ : Absence of the $(\sqrt{3} \times \sqrt{ } 3) \mathrm{R} 30^{\circ}$ Phase. Surf. Sci. 2012, 606, L31-L35.

(47) Kondoh, H.; Nozoye, H. Low-Temperature Ordered Phase of Methylthiolate Monolayers on $\mathrm{Au}(111)$. J. Phys. Chem. B 1999, 103, $2585-2588$.

(48) Maksymovych, P.; Voznyy, O.; Dougherty, D. B.; Sorescu, D. C.; Yates, J. T. Gold Adatom as a Key Structural Component in SelfAssembled Monolayers of Organosulfur Molecules on $\mathrm{Au}(111)$. Prog. Surf. Sci. 2010, 85, 206-240.

(49) Torrelles, X.; Pensa, E.; Cortés, E.; Salvarezza, R.; Carro, P.; Guerrero, C. H.; Ocal, C.; Barrena, E.; Ferrer, S. Solving the Long- 
Standing Controversy of Long-Chain Alkanethiols Surface Structure on $\mathrm{Au}(111)$. J. Phys. Chem. C 2018, 122, 3893-3902.

(50) Liao, S.; Shnidman, Y.; Ulman, A. Adsorption Kinetics of Rigid 4-Mercaptobiphenyls on Gold. J. Am. Chem. Soc. 2000, 122, 36883694. 